\title{
NEW Volkswagen - the MEB shaping the future of integrated e-mobility
}

Christian Senger, Volkswagen AG

This manuscript was not available on completion of this publication. Thank you for your understanding. 